Provided for non-commercial research and education use. Not for reproduction, distribution or commercial use.

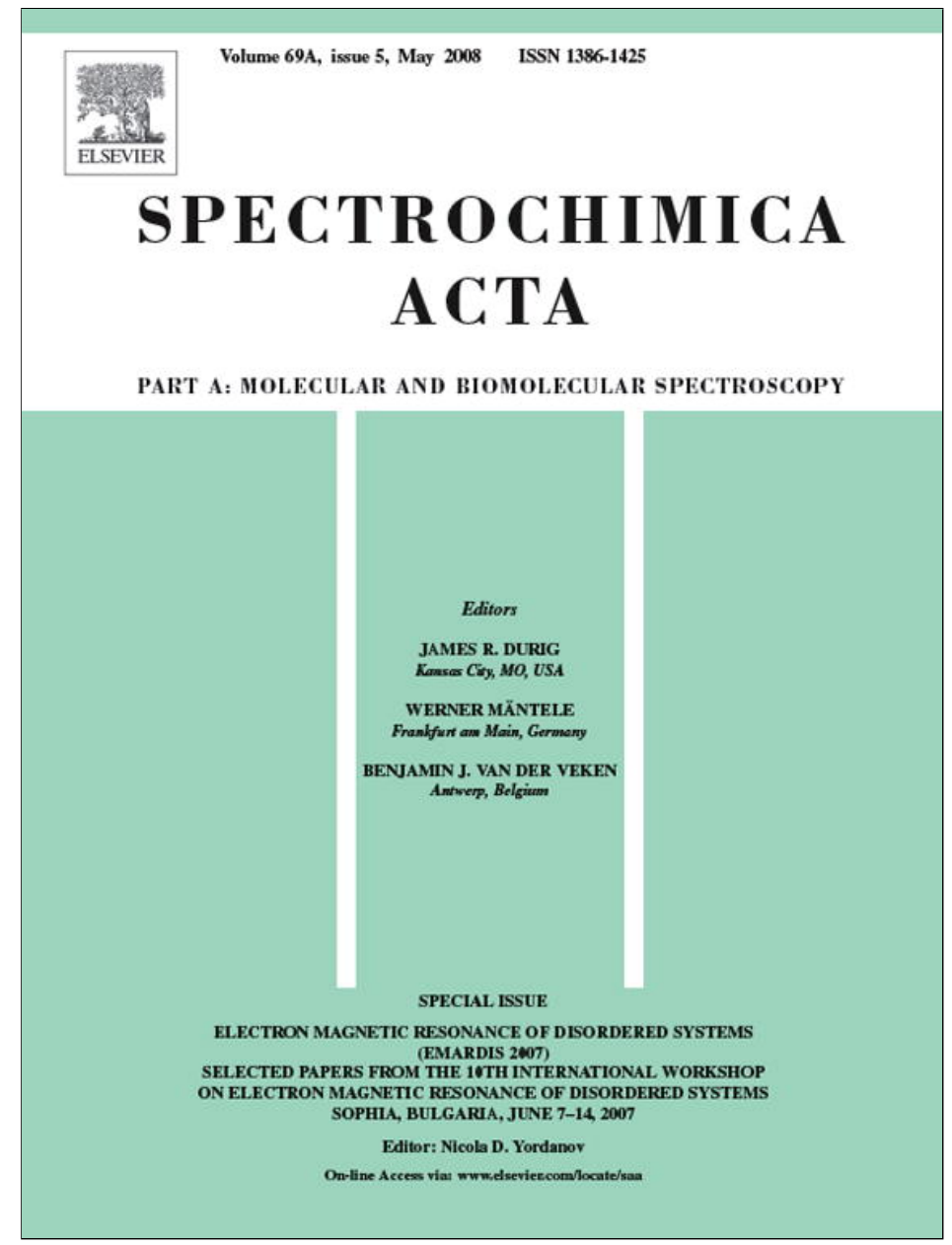

This article appeared in a journal published by Elsevier. The attached copy is furnished to the author for internal non-commercial research and education use, including for instruction at the authors institution and sharing with colleagues.

Other uses, including reproduction and distribution, or selling or licensing copies, or posting to personal, institutional or third party websites are prohibited.

In most cases authors are permitted to post their version of the article (e.g. in Word or Tex form) to their personal website or institutional repository. Authors requiring further information regarding Elsevier's archiving and manuscript policies are encouraged to visit:

http://www.elsevier.com/copyright 


\title{
Effect of temperature on the EPR properties of a rhamnose alkoxy radical: A DFT molecular dynamics study
}

\author{
Ewald Pauwels*, Toon Verstraelen, Michel Waroquier \\ Center for Molecular Modeling, Ghent University, Proeftuinstraat 86, B-9000 Gent, Belgium
}

Received 9 August 2007; accepted 17 September 2007

\begin{abstract}
It has been shown previously that two distinctive variants (called RHop and RO4) exist of the radiation-induced rhamnose alkoxy radical. Density functional theory (DFT) calculations of the electron paramagnetic resonance (EPR) properties were found to be consistent with two separate measurements at different temperatures [E. Pauwels, R. Declerck, V. Van Speybroeck, M. Waroquier, Radiat. Res., in press]. However, the agreement between theory and experiment was only of a qualitative nature, especially for the latter radical. In the present work, it is examined whether this residual difference between theoretical and experimental spectroscopic properties can be explained by explicitly accounting for temperature in DFT calculations. With the aid of ab-initio molecular dynamics, a temperature simulation was conducted of the RO4 variant of the rhamnose alkoxy radical. At several points along the MD trajectory, $g$ and hyperfine tensors were calculated, yielding time (and temperature) dependent mean spectroscopic properties. The effect of including temperature is evaluated but found to be within computational error. (c) 2007 Elsevier B.V. All rights reserved.
\end{abstract}

Keywords: DFT; EPR; Solid state; Alkoxy radical; $\alpha$-Rhamnose; Periodic calculations; Molecular dynamics; Hyperfine coupling; $g$ tensor

\section{Introduction}

One of the species that is commonly generated in irradiated crystalline sugars is an alkoxy radical. These oxygen-centered species readily decay under the influence of temperature or light and are generally considered to be primary radiation products. As such, they have attracted considerable attention. Since sugars have some features that are also present in more complex biomolecules (e.g. the deoxyribose unit in DNA), they effectively present ideal test systems to investigate initial radiation-induced events. Crystalline $\alpha$-L-rhamnose is a representative system in this respect, since both primary oxidation and reduction processes have been identified in this sugar.

The leading technique to examine alkoxy radicals is electron paramagnetic resonance (EPR) spectroscopy at very low temperatures (both irradiation and measurement). The lack of thermal energy limits the conversion of primary radiation products in secondary reactions and this allows thorough spectroscopic characterization. Two EPR studies have been undertaken of the alkoxy radical in rhamnose. Samskog and Lund performed

\footnotetext{
* Corresponding author. Fax: +329264 6697.

E-mail address: ewald.pauwels@UGent.be (E. Pauwels).
}

a Q-band EPR measurement at $77 \mathrm{~K}$ and determined the $g$ tensor, along with two hyperfine coupling constants [2]. In a later study by Budzinski and Box, EPR and ENDOR (electron nuclear double resonance) were used to thoroughly characterize this species at $4.2 \mathrm{~K}$ : the $g$ tensor and seven hyperfine tensors were distinguished [3]. Even though the same radical structure was proposed in both studies, the two experimental data sets are strikingly dissimilar, as noted by other authors [4]. The $77 \mathrm{~K}$ measurement revealed two hyperfine tensors with isotropic couplings of 112 and $39 \mathrm{MHz}$. At $4.2 \mathrm{~K}$ conversely, a myriad of smaller hyperfine interactions was detected along with two major isotropic couplings of 67 and $54 \mathrm{MHz}$. But both studies also differed in the $g$ tensor: a maximum anisotropic $g$ tensor component of 2.0456 was reported by Samskog and Lund, whereas Budzinski and Box found 2.0202.

In a recent theoretical study by the authors a basic reconciliation was made of both EPR studies [1]. The monohydrate crystal of rhamnose was simulated using density functional theory (DFT) in a periodic approach and several reaction steps were examined leading up to alkoxy radical formation. The proposed mechanism is summarized in Fig. 1. Resulting from oxidation, a primary rhamnose cation (PrimCat+) ejects a proton that migrates first to a nearby crystal water and then further on into the lattice. Molecular modeling provided 


\section{PrimCat $^{+}$}

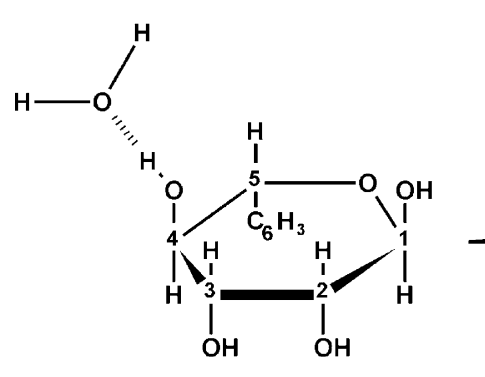

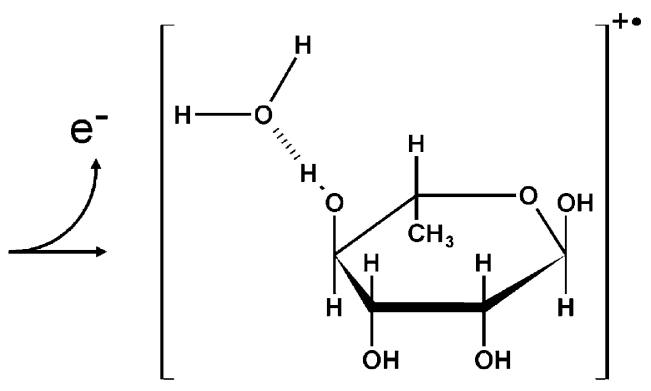<smiles>O</smiles><smiles>CCCCO[C@H]1C(O)[C@H](O)[C@H](O)[C@H]1O</smiles><smiles>CC1CC(O)C(O)C(O)OC1C=O</smiles>

RO4

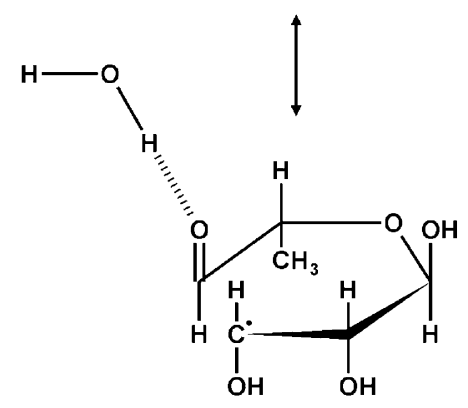

RHop

Fig. 1. Atom numbering scheme and summary of reaction steps leading to alkoxy radical formation in $\alpha$-L-rhamnose. Oxygens and hydrogens are numbered according to the carbon to which they are bound.

evidence that this migration is in fact a proton hopping process - similar to the Grotthuss mechanism in solutions [5] - along an 'infinite' hydrogen bond chain parallel to the $\langle b\rangle$ axis of the crystal. The resultant of this proton removal is an alkoxy radical (dubbed RHop), for which calculated EPR properties were in close agreement with the $4.2 \mathrm{~K}$ EPR measurements. Yet, the subsequent proton hops alter the hydrogen bonds, also in the vicinity of the radical. The initial state of the H-bonds can be restored, by rotating several hydroxyl groups and crystal waters to their original orientation in the undamaged crystal. In this way the more stable RO4 variant of the alkoxy radical was obtained and calculated EPR properties were in agreement with the $77 \mathrm{~K}$ EPR measurements. Hence, the main difference between the two radicals observed in the experiments was not the radical structure itself, but rather its environment.

Yet, although the theoretical EPR predictions for RO4 and RHop succeeded in clarifying the differentiation between the 4.2 and $77 \mathrm{~K}$ measurements on a qualitative level, the reproduction on the quantitative level of the individual hyperfine and $g$ tensor values with their experimental counterparts could be subject to some improvement. In particular for $\mathrm{RO} 4$, relatively large differences were found [1] for the maximum anisotropic $g$ tensor component (2.0263 instead of 2.0456 experimentally) and - to a smaller extent - for the hyperfine couplings (50.4 and $87.1 \mathrm{MHz}$ instead of 39.2 and $112.1 \mathrm{MHz}$ ). One could ascribe these residual differences between theory and experiment to temperature effects not taken into account in the theoretical description of the radical system. Static calculations simulate the system at a temperature of $0 \mathrm{~K}$. One could argue that such an approach is acceptable for a comparison between the calculated spectroscopic properties of RHop and the $4.2 \mathrm{~K}$ measurements, but is insufficient when evaluating the theoretical EPR properties of RO4 with regard to those measured at a higher temperature of $77 \mathrm{~K}$. In this work, the validity of this statement is assessed and calculations are presented on the RO4 alkoxy radical in which temperature is duly accounted for. To this end, molecular dynamics simulations have been performed of the radical structure at $77 \mathrm{~K}$, adopting a periodic approach to include the molecular environment. 


\section{Computational details}

In the previous study [1], the structure of $\alpha$-L-rhamnose monohydrate [6] was consistently described in a periodic approach, exploiting the translational symmetry of the crystalline state. The original unit cell of the crystal was doubled in all directions, to ensure that the radical was well separated from its periodic images. However, the results of the calculations within this $\langle 2 a 2 b 2 c\rangle$ supercell - containing 416 atoms were found to be virtually on the same level as within an $\langle a 2 b c\rangle$ cell, obtained by only doubling the unit cell in the $\langle b\rangle$ direction. Therefore, the latter supercell was chosen in the present work as it considerably reduces the computational burden. An initial structure for the radical was obtained by removing the HO4 hydroxy proton from the model space (see Fig. 1 for the atom numbering scheme).

All periodic calculations were performed using the ab-initio Car-Parrinello approach [7] as implemented in the CPMD software package [8]. The BP86 gradient-corrected density functional [9] was used, together with a plane wave basis set (cutoff $25 \mathrm{Ry}$ ) and ultra-soft pseudopotentials of the Vanderbilt type to describe the electron-ion interaction [10]. First, the global minimum of the radical within the $\langle a 2 b c\rangle$ supercell was determined in a (static) geometry optimization. No constraints were imposed on any of the atoms in the supercell. Molecular dynamics simulations were then initiated starting from the completely optimized structure, with a simulation temperature of $77 \mathrm{~K}$. Initial calculations were performed to quickly equilibrate the temperature of the system by rescaling ionic velocities whenever the instantaneous temperature differed more than $50 \mathrm{~K}$ from the target temperature (1000 time steps). Subsequently, a Nosé-Hoover thermostat was activated for the ionic and electronic systems. A characteristic frequency of $3000 \mathrm{~cm}^{-1}$ was chosen for the ionic thermostat, with a target temperature of $77 \mathrm{~K}$. The electron thermostat was set to a frequency of $10,000 \mathrm{~cm}^{-1}$ and an average target kinetic energy of 0.012430 a.u. was determined for the electronic system from separate NVE molecular dynamics simulations. The MD timestep was 5 a.u. ( $0.12 \mathrm{fs}$ ) and the fictitious electronic mass was set to 400 a.u. The Nosé-Hoover run was equilibrated for 2000 time steps, after which 8000 further steps were considered as production quality, amounting to about $0.97 \mathrm{ps}$.

To determine the temperature dependence of the hyperfine and $g$ tensors, EPR properties were calculated for 200 sampled snapshots along the resulting MD trajectory of 8000 time steps (one snapshot every 40 time steps). Spectroscopic parameters were determined consistent with the computational protocol adopted in the previous work [1]. For each snapshot, a cluster was cut out of the periodic system to contain the radical and all the molecules that are hydrogen bound to it (seven rhamnose and eight water molecules). Hyperfine tensors were calculated with the aid of the Gaussian03 software suite [11], using the B3LYP functional [12] and a 6-311G** basis set [13] for all atoms within the cluster. However, this level of theory is prohibitively expensive from a computational point of view for the calculation of $g$ tensors. Consequently, the $6-311 \mathrm{G}^{* *}$ basis set was only maintained for the atoms of the central radical along with those of two nearby water molecules. The other atoms of the cluster were still included in the calculation at the B3LYP level of theory, but were considered at the much smaller 3-21G basis set [14].

\section{Results and discussion}

Since the smaller $\langle a 2 b c\rangle$ supercell was adopted in the present work, the validity of this choice was evaluated by benchmarking with previous calculations using a $\langle 2 a 2 b 2 c\rangle$ supercell approach [1]. The $\langle a 2 b c\rangle$ optimized structure was compared with the part of the $\langle 2 a 2 b 2 c\rangle$ supercell matching the size of the smaller supercell and containing the radical site. This resulted in an RMSD of $0.009 \AA$, indicating that both structures are essentially similar. EPR properties were also calculated for the optimized $\langle a 2 b c\rangle$ supercell and compared with the results obtained earlier. An overview of calculated as well as measured EPR properties is presented in Table 1. Comparing sections (b) and (c), it is clear that the supercell size reduction does not really have a noticeable effect on the calculated EPR properties of the alkoxy radical. A slight change is only noticed in the H4 isotropic coupling, which rises to $90.4 \mathrm{MHz}$ and is now somewhat closer to the actual experimental value (see Table 1(a)). Yet, structurally and electronically it appears that a $\langle a 2 b c\rangle$ supercell is virtually identical to a $\langle 2 a 2 b 2 c\rangle$ one, endorsing the chosen computational approach.

Exploring the geometrical changes of the radical and its environment during the $77 \mathrm{~K}$ molecular dynamics run, only small fluctuations from the statically optimized $\langle a 2 b c\rangle$ structure were registered. The mean $77 \mathrm{~K}$ geometry - obtained by averaging all 8000 geometries in the MD run - differs by only $0.008 \AA$ from the static geometry. On the other hand, large variations are noticed for the EPR properties calculated at 200 snapshots along the trajectory. This is illustrated in Fig. 2, where

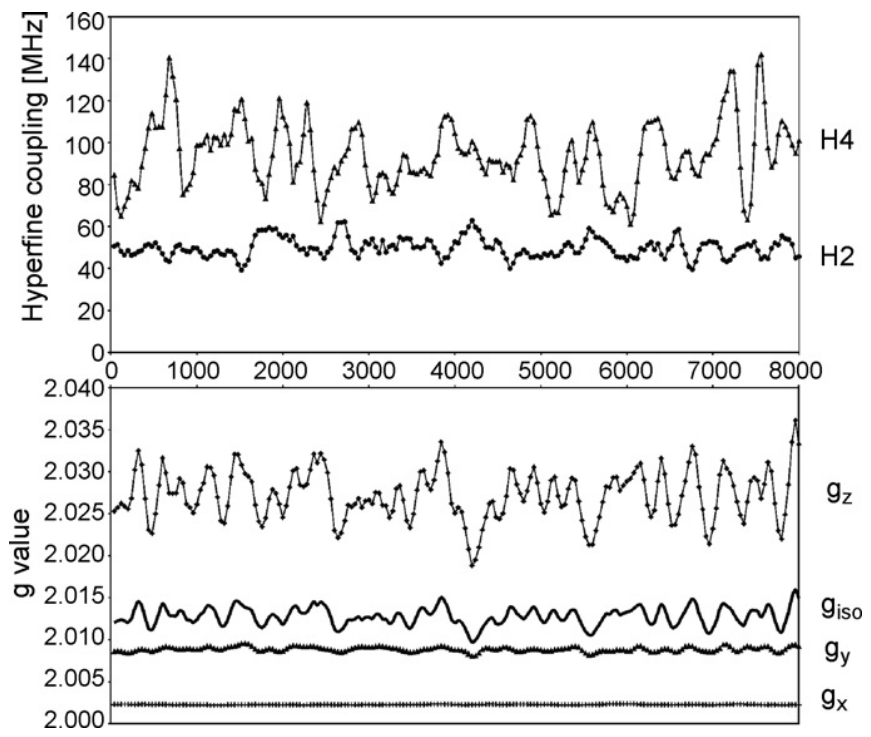

Fig. 2. Isotropic hyperfine couplings (in $\mathrm{MHz}$ ) and (an)isotropic $g$ tensor values as a function of time step $(0.12 \mathrm{fs})$ in the molecular dynamics simulation. $g_{x}$, $g_{y}$ and $g_{z}$ refer to the minor, intermediate and maximum anisotropic $g$ tensor components, respectively. 
Table 1

Overview of calculated and measured EPR data

\begin{tabular}{|c|c|c|c|c|c|c|}
\hline \multirow{2}{*}{\multicolumn{2}{|c|}{$\frac{A_{\text {iso }} / g_{\text {iso }}}{\text { (a) Experimental EPR properties }}{ }^{\mathrm{a}}$}} & \multirow[t]{2}{*}{$A_{\text {aniso }} / g_{\text {aniso }}$} & \multicolumn{3}{|c|}{ Direction cosines versus $\langle a * b c\rangle$} & \multirow[t]{2}{*}{$\Psi$} \\
\hline & & & & & & \\
\hline $\mathrm{H} 2$ & 39.2 & & & & & \\
\hline $\mathrm{H} 4$ & 112.1 & & & & & \\
\hline & & 2.0032 & -0.020 & -0.982 & 0.189 & \\
\hline $\mathrm{g}$ & 2.0184 & 2.0064 & -0.698 & 0.149 & 0.700 & \\
\hline & & 2.0456 & -0.716 & -0.118 & -0.688 & \\
\hline \multicolumn{7}{|c|}{ (b) Static EPR calculation on optimized $\langle 2 a 2 b 2 c\rangle$ supercell $^{\mathrm{b}}$} \\
\hline & & -3.5 & 0.692 & -0.528 & -0.492 & \\
\hline \multirow[t]{3}{*}{$\mathrm{H} 2$} & 50.4 & -0.3 & 0.579 & -0.001 & 0.815 & \\
\hline & & 3.8 & 0.431 & 0.849 & -0.305 & \\
\hline & & -5.9 & 0.443 & 0.881 & 0.163 & \\
\hline \multirow[t]{3}{*}{$\mathrm{H} 4$} & 87.1 & -4.6 & -0.209 & -0.075 & 0.975 & \\
\hline & & 10.5 & 0.872 & -0.466 & 0.151 & \\
\hline & & 2.0023 & -0.316 & -0.839 & 0.443 & 24 \\
\hline \multirow[t]{2}{*}{$g$} & 2.0125 & 2.0087 & -0.547 & 0.542 & 0.637 & 25 \\
\hline & & 2.0263 & -0.775 & -0.041 & -0.631 & 6 \\
\hline \multicolumn{7}{|c|}{ (c) Static EPR calculation on optimized $\langle a 2 b c\rangle$ supercell } \\
\hline & & -3.4 & 0.695 & -0.523 & -0.494 & \\
\hline \multirow[t]{3}{*}{$\mathrm{H} 2$} & 49.6 & -0.2 & 0.549 & -0.059 & 0.834 & \\
\hline & & 3.6 & 0.465 & 0.851 & -0.246 & \\
\hline & & -6.1 & 0.448 & 0.882 & 0.146 & \\
\hline \multirow[t]{3}{*}{$\mathrm{H} 4$} & 90.4 & -4.7 & -0.198 & -0.061 & 0.978 & \\
\hline & & 10.8 & 0.872 & -0.468 & 0.148 & \\
\hline & & 2.0023 & -0.314 & -0.872 & 0.375 & 21 \\
\hline \multirow[t]{2}{*}{$g$} & 2.0126 & 2.0088 & -0.572 & 0.489 & 0.659 & 21 \\
\hline & & 2.0267 & -0.758 & -0.008 & -0.652 & 7 \\
\hline \multicolumn{7}{|c|}{ (d) Dynamic EPR calculation on $\langle a 2 b c\rangle$ supercell at $77 \mathrm{~K}$} \\
\hline & & -3.4 & 0.692 & -0.526 & -0.495 & \\
\hline \multirow[t]{3}{*}{$\mathrm{H} 2$} & 49.9 & -0.2 & 0.551 & -0.058 & 0.832 & \\
\hline & & 3.6 & 0.467 & 0.848 & -0.250 & \\
\hline & & -6.2 & 0.454 & 0.884 & 0.108 & \\
\hline \multirow[t]{3}{*}{$\mathrm{H} 4$} & 94.0 & -4.7 & -0.184 & -0.025 & 0.983 & \\
\hline & & 10.9 & 0.872 & -0.466 & 0.152 & \\
\hline & & 2.0023 & -0.308 & -0.877 & 0.369 & 20 \\
\hline \multirow[t]{2}{*}{$\mathrm{g}$} & 2.0128 & 2.0089 & -0.581 & 0.480 & 0.657 & 20 \\
\hline & & 2.0272 & -0.753 & -0.012 & -0.658 & 7 \\
\hline \multicolumn{7}{|c|}{ (e) Static EPR calculation on optimized cluster ${ }^{\mathrm{c}}$} \\
\hline & & -3.0 & 0.705 & -0.521 & -0.481 & \\
\hline \multirow[t]{3}{*}{$\mathrm{H} 2$} & 40.5 & -0.3 & 0.488 & -0.136 & 0.862 & \\
\hline & & 3.3 & 0.515 & 0.843 & -0.159 & \\
\hline & & -6.5 & 0.470 & 0.870 & -0.151 & \\
\hline \multirow[t]{3}{*}{$\mathrm{H} 4$} & 100.4 & -4.4 & -0.152 & 0.249 & 0.956 & \\
\hline & & 11.0 & 0.870 & -0.426 & 0.249 & \\
\hline & & 2.0022 & -0.251 & -0.885 & 0.392 & 19 \\
\hline \multirow[t]{2}{*}{$g$} & 2.0189 & 2.0090 & -0.690 & 0.448 & 0.569 & 19 \\
\hline & & 2.0456 & -0.679 & -0.128 & -0.723 & 3 \\
\hline
\end{tabular}

Hyperfine couplings are presented in MHz. The $\Psi$ angle (in degrees) indexes the deviation in orientation between the calculated eigenvectors and their experimental counterparts.

${ }^{a}$ Ref. [2].

b Ref. [1].

c Ref. [15].

the isotropic hyperfine and (anisotropic) $g$ tensor values are plotted as a function of time step in the molecular dynamics simulation. Especially the $\mathrm{H} 4$ hyperfine coupling and the $g_{z}$ (major) anisotropic $g$ tensor component vary significantly. This reveals that the EPR properties of the rhamnose alkoxy radical are quite sensitive to even minute changes in the geometry.
Based on the 200 samples of the EPR properties along the trajectory, it is now possible to calculate the thermal average of these properties. For isotropic values, this can simply be performed by averaging the results of the 200 snapshot calculations. To determine the anisotropic components and the corresponding eigenvectors, however, it is necessary to first calculate the mean, non-diagonalized $g$ - or hyperfine matrices and then diagonalize 
this matrix. The resulting dynamic EPR properties are presented in Table 1(d). Overall, the eigenvectors and anisotropic hyperfine couplings are least affected by the dynamics, since they are nearly identical to those in Table 1(c). For the $\mathrm{H} 4$ isotropic hyperfine coupling, the isotropic $g$ value and the maximum anisotropic $g$ component $\left(g_{z}\right)$, the differences are small, but discernible: the $\mathrm{H} 4$ coupling increases to $94 \mathrm{MHz}$ and $g_{z}$ rises by about $450 \mathrm{ppm}$ (to 2.0272). Although these changes do seem to enhance the agreement between theory and experiment, the effect is only minor and too small to classify it as an improvement. In other words, the temperature effect does not really account for the remaining discrepancies between theoretical and experimental EPR properties of the RO4 alkoxy radical. Of course, the time scale over which the thermal average has been taken $(\sim 1 \mathrm{ps})$ is relatively limited and the effect of molecular motions with longer periods is not accounted for. One could improve this by increasing the time step and/or total simulation time in the MD, although calculations at the nanosecond scale are prohibitively expensive at the ab-initio level and still far below the typical time scale of the CW-EPR experiment ( $\mu s)$.

Apart from the previous conclusions, it is worth noticing that the calculated EPR properties for rhamnose, reported earlier by some of the authors [15], are in much better agreement with experiment than the values determined in the current MD simulation. In that study, the RO4 alkoxy radical has been investigated within a cluster approach. For comparison, these results are summarized in Table 1(e). Although large similarities exist in the reproduction of the eigenvectors and anisotropic couplings, the isotropic hyperfine splittings vary substantially (almost by $9 \mathrm{MHz}$ as compared to the dynamic calculation) and are in closer agreement with experiment. Even more spectacularly, the $g_{z}$ anisotropic component exactly equals the experimental value of 2.0456! And yet, from a theoretical point of view, several aspects in the earlier approach were essentially inferior to the methodology adopted in the current work:

(i) Because a cluster approach was followed for the geometry optimization, the molecular environment of the radical was finite, contrary to the current periodic approach. But more importantly, constraints were imposed during optimization on all atoms except those of the central radical in the cluster. This effectively prevented any relaxation in the molecular environment of the radical away from the ideal crystal structure. Nevertheless, the resulting cluster optimized geometry is very similar to the structure of the $\langle a 2 b c\rangle$ supercell optimization in the present work. The RMSD with the cluster cut out of the periodic calculation - which was used for the calculation of the EPR properties - amounts only to $0.005 \AA$. Hence, since the same computational protocol was used in [15] and the current work to determine the hyperfine coupling constants (B3LYP functional with 6-311G** basis set for all atoms of the cluster), it must be concluded that the differences in (isotropic) hyperfine couplings between Table 1(c) and (e) are solely due to slight geometrical changes. This is consistent with the observation that in Fig. 2, the EPR properties can vary significantly to even minute changes in the geometry (see above).

(ii) In the previous work [15], a different computational protocol was used for the calculation of the $g$ tensor. A 'single molecule' approach was followed and $g$ tensor properties were calculated solely on the radical itself, neglecting any interaction with its molecular environment. Although this approach seemed to work amazingly well in the case of the $g_{z}$ component, in retrospect it is probable that it did so for the wrong reasons. A $g$ tensor calculation of a single rhamnose alkoxy radical, without taking into account the molecular environment of the radical, showed in principle the same values for the RO4 and RHop radical variants. The incorporation of surrounding molecules, as in reference [1], yields $g$ tensors that are distinct for these two species, although in worse quantitative agreement with experiment. Hence, it is conceivable that error cancellation effects in the single molecule calculations have coincidentally resulted in an identical reproduction of the measured $g_{z}$ anisotropic $g$ tensor component. But, for the moment it remains unclear what the real origin is of the apparent discrepancy between the current, time-averaged $g$ tensor calculations and the experiment. This paper shows that temperature effects are too moderate to remove this failure.

Regardless, the molecular dynamics simulation reveals that some large variations of the relevant spectroscopic properties take place (see Fig. 2). The H4 coupling may vary from 60 to $140 \mathrm{MHz}$ and the experimental value of $112 \mathrm{MHz}$ belongs to that interval. These variations are the result of geometry changes generated by the MD simulations. It could be interesting to search for a correlation between the calculated EPR properties and geometry. For this purpose, linear regression analyses were performed with the main EPR properties as dependent variables $\left(g_{z}\right.$ value, $\mathrm{H} 2$ and $\mathrm{H} 4$ isotropic couplings), and all internal coordinates of the radical and nearby water molecules as explanatory variables ( 27 bond lengths, 26 bond angles and 25 torsional angles). This is actually a limited set of structural parameters since other coordinates could be chosen involving more molecules of the supercell. Nevertheless, the subset proved more than sufficient and, moreover, a variance of up to 0.8 was readily obtained for all spectroscopic properties by including only eight internal coordinates in the regression. However, it is difficult to understand the variation of the hyperfine or $g$ tensor values in terms of all these parameters from a chemical point of view. Hence, the parameter set was further reduced in an attempt to explain the variance of the EPR properties by considering only one or two of the dominant predictor variables. Fig. 3 plots the variance of the $g_{z}, \mathrm{H} 2$ and $\mathrm{H} 4$ isotropic coupling values with respect to the selected variables.

(i) $g_{z}$ value

The variation of $g_{z}$ was found to mainly depend on two geometric coordinates: the $\mathrm{C} 3-\mathrm{C} 4$ bond length in the alkoxy radical, and the length of a hydrogen bond between oxygen $\mathrm{O} 4$ and one of the protons of a crystal water. A lin- 

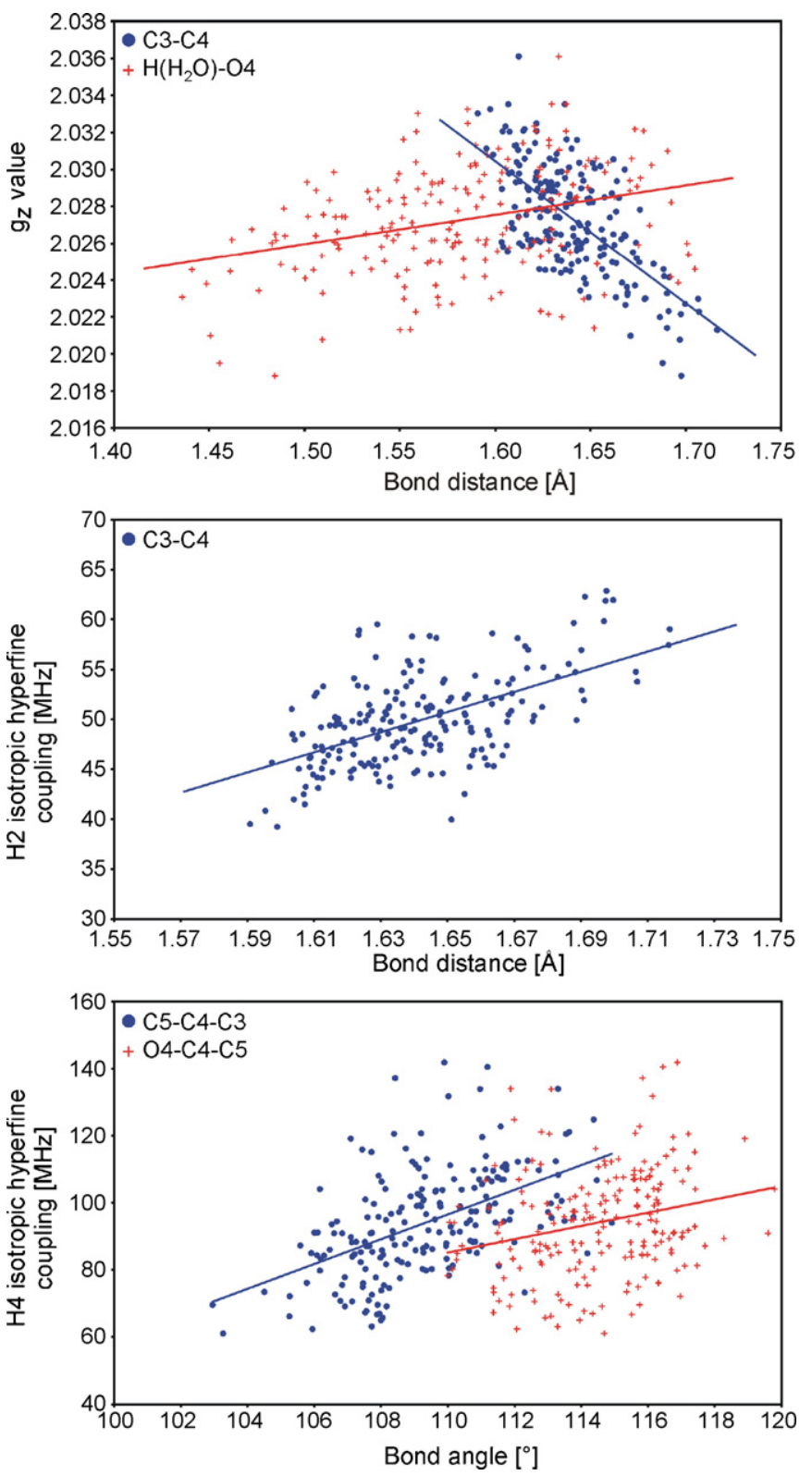

Fig. 3. Dependence of $g_{z}$ and isotropic $\mathrm{H} 2 / \mathrm{H} 4$ coupling values with respect to several internal coordinates within the rhamnose alkoxy radical. $\mathrm{H}\left(\mathrm{H}_{2} \mathrm{O}\right)$ refers to one of the protons within the $\langle a 2 b c\rangle$ supercell that is hydrogen bound to $\mathrm{O} 4$.

ear regression model including only these two explanatory variables already accounted for $56 \%$ of $g_{z}$ variance. As is apparent in Fig. 3, the inverse correlation with $\mathrm{C} 3-\mathrm{C} 4$ is quite strong $(-0.67)$, which can be attributed to the existence of resonance in both RHop and RO4 variants of the rhamnose alkoxy radical. In the alternative resonance structure, the unpaired electron is mainly localized on C3 instead of on $\mathrm{O} 4$, as is illustrated in the bottom of Fig. 1. The larger the $\mathrm{C} 3-\mathrm{C} 4$ bond length, the more this resonance structure will contribute and the less spin density will be localized on $\mathrm{O} 4$, giving rise to a decrease in $g_{z}$. A similar interpretation is valid for the $\mathrm{H}\left(\mathrm{H}_{2} \mathrm{O}\right)-\mathrm{O} 4$ hydrogen bond length. As this hydrogen bond elongates, the spin density will become more isolated on $\mathrm{O} 4$ and $g_{z}$ will increase. (ii) $\mathrm{H} 2$

C3-C4 bond elongation also explains the variation in the $\mathrm{H} 2$ hyperfine coupling to a large extent (correlation 0.57 ). This is immediately clear when considering the resonance structure in Fig. 1. The more spin density is localized on $\mathrm{C} 3$, the larger the isotropic $\mathrm{H} 2$ hyperfine coupling will be. In several previous works $[1,3]$ this relation was already suggested.

(iii) $\mathrm{H} 4$

The variation of the isotropic $\mathrm{H} 4$ coupling proved quite difficult to interpret with only a limited number of structural parameters. The consideration of the two most important internal coordinates led to an explained variance of only 0.38. Apparently, the coupling is sensitive to changes in the bond angles of the rhamnose pyranose ring (C5-C4-C3) and the relative position of the $\mathrm{C} 4-\mathrm{O} 4$ axis with respect to the ring (reflected by the $\mathrm{O} 4-\mathrm{C} 4-\mathrm{C} 5$ bond angle). The $\mathrm{H} 4$ coupling does not depend on the $\mathrm{C} 3-\mathrm{C} 4$ bond distance, however. For both resonance structures of Fig. 1, $\mathrm{H} 4$ remains in a beta position with respect to the main site of unpaired spin density.

The importance of resonance in the rhamnose alkoxy radical is further exemplified in a vibrational analysis of the periodic molecular system. In a molecular dynamics simulation, atomic positions as well as velocities are determined. By using the latter and calculating the Fourier transform (FFT) of the velocity autocorrelation function, a mass-weighted power spectrum can be obtained yielding vibrational frequencies. The resulting spectra at $77 \mathrm{~K}$ are shown in Fig. 4 for the entire supercell (dashed line) and only for velocity components of atoms belonging to the radical (solid line). In principle, these spectra could be compared with infrared measurements. However, because changes in dipole moment were not determined throughout the MD simulation, the computed intensities are not really meaningful (they could well be IR-silent for instance). Nevertheless the calculated spectra do reveal what kind of vibrational modes are present in the system. Particular attention was paid to modes that only occur

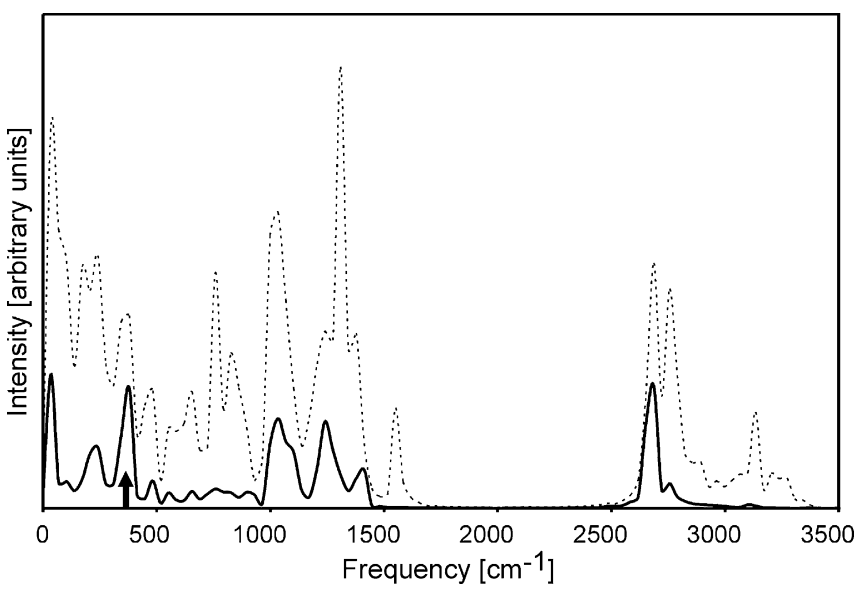

Fig. 4. Vibrational frequency spectrum for the radical (black line) and all atoms of the $\langle a 2 b c\rangle$ supercell (dashed line). 
in the radical and not in the 'undamaged' rhamnose molecules within the supercell, and vice versa. It is clear from the figure that the spectrum for the entire supercell reveals several peaks (e.g. at $1550,3135 \mathrm{~cm}^{-1}$ ) that are not present in the spectrum of the radical. These modes could be attributed to the relative movement of crystal water. The majority of peaks in the spectrum for the radical are also present in the spectrum for the entire supercell. Only one mode is predominantly due to vibrations of the radical: the peak at $378 \mathrm{~cm}^{-1}$ (indicated by an arrow in Fig. 4). Structural analysis reveals that it specifically involves strong C3-C4 bond length vibrations, combined with less intense rotational modes of the alkoxy pyranose ring. Hence, one could state that for this low vibrational mode, the RO4 rhamnose alkoxy radical rapidly switches between both resonance structures as depicted in Fig. 1.

\section{Conclusions}

In this article, a temperature simulation of the RO4 variant of the rhamnose alkoxy radical was conducted with the aid of ab-initio molecular dynamics. It was examined whether these temperature simulations could account for the residual difference between theoretical and experimental spectroscopic properties. At several points along the MD trajectory, $g$ and hyperfine tensors were calculated and averaged, resulting in temperature dependent mean values. The effect of including temperature was found to be within computational error and no improvement was obtained between theory and experiment.

Additionally, the dynamics of the alkoxy radical were further explored. Using linear regression models, the variance of the $g_{z}$ value, $\mathrm{H} 2$ and $\mathrm{H} 4$ hyperfine couplings was considered in relation to several geometric internal coordinates of the radical structure. It was found that $g_{z}$ and $\mathrm{H} 2$ are considerably affected by the $\mathrm{C} 3-\mathrm{C} 4$ bond distance. This could be ascribed to the existence of a resonance structure of the alkoxy radical, through which spin density gets delocalized on the $\mathrm{C} 3$ carbon center. In a vibrational analysis of the molecular dynamics, a low frequency mode was furthermore identified with $\mathrm{C} 3-\mathrm{C} 4$ bond vibration as main characteristic. Apparently, the structure of the RO4 rhamnose alkoxy radical is alternating between resonance structures at a finite temperature.

\section{Acknowledgements}

This work is supported by the Fund for Scientific Research Flanders (FWO). The authors kindly acknowledge Dr. R. Raymaekers for assisting with the linear regression analyses.

\section{References}

[1] E. Pauwels, R. Declerck, V. Van Speybroeck, M. Waroquier, Radiat. Res, in press.

[2] P.O. Samskog, A. Lund, Chem. Phys. Lett. 75 (1980) 525-527.

[3] E.E. Budzinski, H.C. Box, J. Chem. Phys. 82 (1985) 3487-3490.

[4] E. Sagstuen, M. Lindgren, A. Lund, Radiat. Res. 128 (1991) 235-242.

[5] C.J.T. de Grotthuss, Ann. Chim. 58 (1806) 54-74; C.J.T. de Grotthuss, Biochim. Biophys. Acta 1757 (2006) 871-875 (English translation by R. Pomès);

N. Agmon, Chem. Phys. Lett. 244 (1995) 456-462.

[6] S. Takagi, G.A. Jeffrey, Acta Cryst. B 34 (1978) 2551-2555.

[7] P. Car, M. Parrinello, Phys. Rev. Lett. 55 (1985) 2471-2474.

[8] CPMD V3.11 Copyright IBM Corp. 1990-2006, Copyright MPI fuer Festkoerperforschung Stuttgart 1997-2001.

[9] J.P. Perdew, Phys. Rev. B 33 (1986) 8822-8824; A.D. Becke, J. Chem. Phys. 96 (1992) 2155-2160.

[10] D. Vanderbilt, Phys. Rev. B 41 (1990) 7892-7895.

[11] Gaussian 03, Revision B.03, M.J. Frisch, G.W. Trucks, H.B. Schlegel, G.E. Scuseria, M.A. Robb, J.R. Cheeseman, J.A. Montgomery, Jr., T. Vreven, K.N. Kudin, J.C. Burant, J.M. Millam, S.S. Iyengar, J. Tomasi, V. Barone, B. Mennucci, M. Cossi, G. Scalmani, N. Rega, G.A. Petersson, H. Nakatsuji, M. Hada, M. Ehara, K. Toyota, R. Fukuda, J. Hasegawa, M. Ishida, T. Nakajima, Y. Honda, O. Kitao, H. Nakai, M. Klene, X. Li, J. E. Knox, H.P. Hratchian, J.B. Cross, C. Adamo, J. Jaramillo, R. Gomperts, R.E. Stratmann, O. Yazyev, A. J. Austin, R. Cammi, C. Pomelli, J.W. Ochterski, P.Y. Ayala, K. Morokuma, G.A. Voth, P. Salvador, J.J. Dannenberg, V.G. Zakrzewski, S. Dapprich, A.D. Daniels, M.C. Strain, O. Farkas, D.K. Malick, A.D. Rabuck, K. Raghavachari, J.B. Foresman, J.V. Ortiz, Q. Cui, A.G. Baboul, S. Clifford, J. Cioslowski, B.B. Stefanov, G. Liu, A. Liashenko, P. Piskorz, I. Komaromi, R.L. Martin, D.J. Fox, T. Keith, M.A. Al-Laham, C.Y. Peng, A. Nanayakkara, M. Challacombe, P.M.W. Gill, B. Johnson, W. Chen, M.W. Wong, C. Gonzalez, J.A. Pople, Gaussian, Inc., Wallingford, CT, 2004.

[12] A.D. Becke, J. Chem. Phys. 104 (1996) 1040-1046.

[13] R. Krishnan, J.S. Binkley, R. Seeger, J.A. Pople, J. Chem. Phys. 72 (1980) 650-654;

A.D. McLean, G.S. Chandler, J. Chem. Phys. 72 (1980) 5639-5648.

[14] J.S. Binkley, J.A. Pople, W.J. Hehre, J. Am. Chem. Soc. 102 (1980) 939-947;

M.S. Gordon, J.S. Binkley, J.A. Pople, W.J. Pietro, W.J. Hehre, J. Am. Chem. Soc. 104 (1982) 2797-2803.

[15] E. Pauwels, V. Van Speybroeck, M. Waroquier, J. Phys. Chem. A 110 (2006) 6504-6513. 